\title{
Erratum: Spontaneous change in trajectory patterns of a self-propelled oil droplet at the air-surfactant solution interface [Phys. Rev. E 91, 032406 (2015)]
}

\author{
Shinpei Tanaka, Yoshimi Sogabe, and Satoshi Nakata
}

(Received 4 October 2018; published 30 October 2018)

DOI: 10.1103/PhysRevE.98.049903

In Fig. 4(b), the $y$ axis should be labeled as $a\left(\mathrm{~mm} / \mathrm{s}^{2}\right)$. The peak at $f=1.0(1 / \mathrm{s})$ shown in Figs. 8 and 9 in the paper was found to be an artifact. It was created during the image extraction process from compressed movies. In addition, there was a
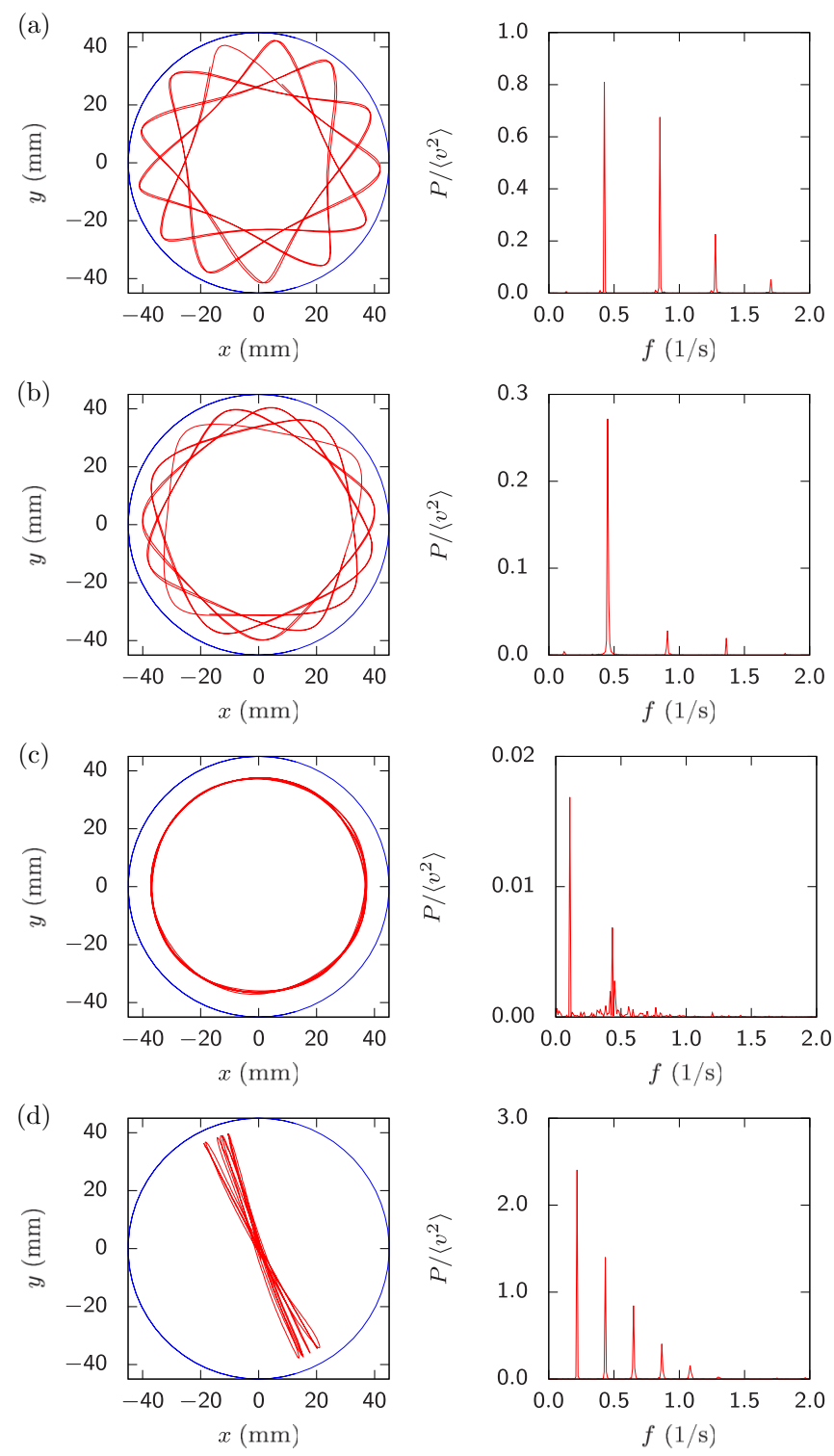

FIG. 8. Experimentally observed global trajectory-pattern transitions (left column) seen in a sample: $10 \mu 1$ ethyl salicylate droplet in a $70 \mathrm{mM}, 30 \mathrm{ml}$ SDS solution with their normalized power spectra of $v-\langle v\rangle$ (right column). The elapsed time $t$ is as follows: (a) 22, (b) 59, (c) 87, and (d) $131 \mathrm{~min}$. Each trajectory was recorded for $1 \mathrm{~min}$. 

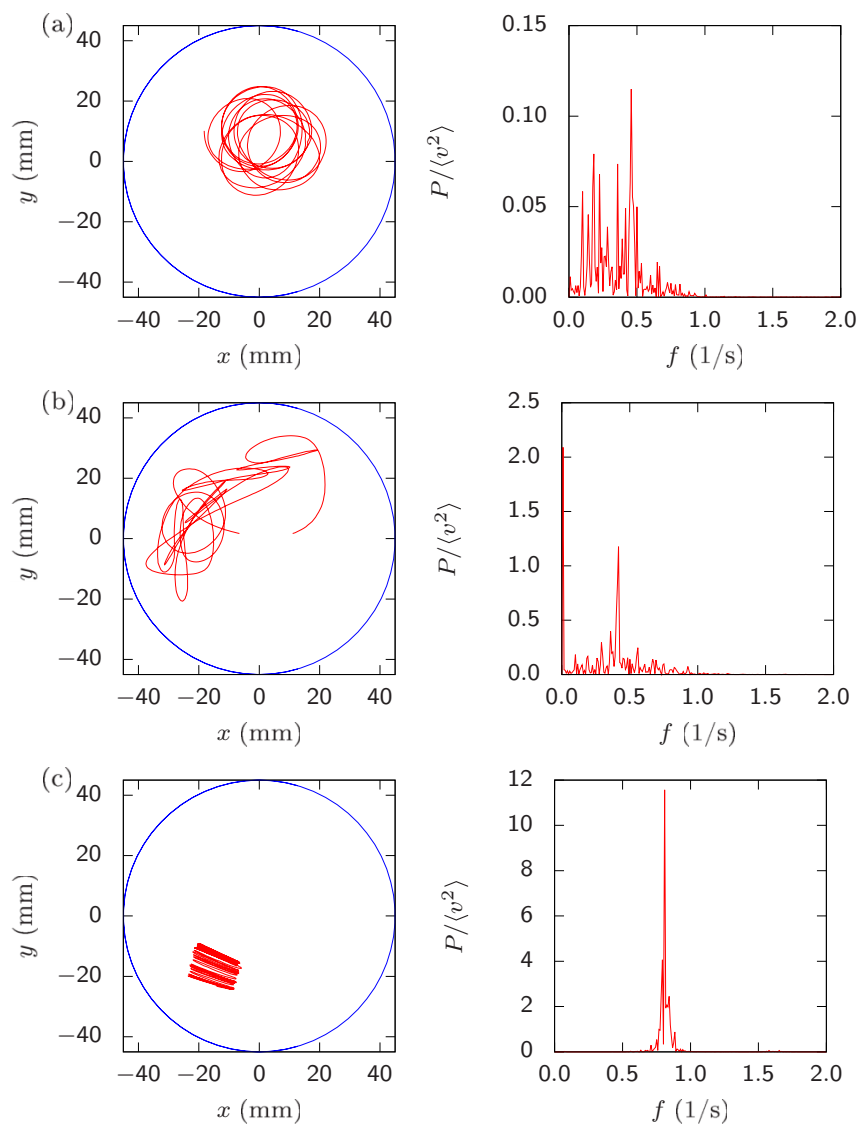

FIG. 9. Experimentally observed local trajectory patterns (left column) seen in the same sample shown in Fig. 8 and their normalized power spectra of $v-\langle v\rangle$ (right column). The elapsed time $t$ is as follows: (a) 156, (b) 168, and (c) 192 min. Each trajectory was recorded for $1 \mathrm{~min}$.

mistake in the normalization of the power. The correct analysis changes, respectively, Figs. 8 and 9 in the paper to Figs. 8 and 9 shown here.

As a result, "the basic oscillation" mentioned in Sec. III A does not need to be introduced. Therefore Sec. III B 3, including Fig. 12, is not needed. Equation (9) should be

$$
\vec{f}_{d}=g(r) a_{d} \vec{v} / v, \quad g(r)= \begin{cases}1, & r \leqslant r_{c}, \\ \frac{b\left(r-r_{c}\right)+(R-r)}{R-r_{c}}, & r_{c}<r \leqslant r_{0}, \\ 0, & r_{0}<r .\end{cases}
$$

Figure 14 in the paper is replaced by Fig. 14 here where the simulation was performed using Eq. (9) above. Figure 13 in the paper was replaced by the result shown in Fig. 13 here, obtained using Figs. 14(a) and 14(c) shown here. As already mentioned in the paper, the basic oscillation does not play any significant role, thus the conclusions in the paper do not change.
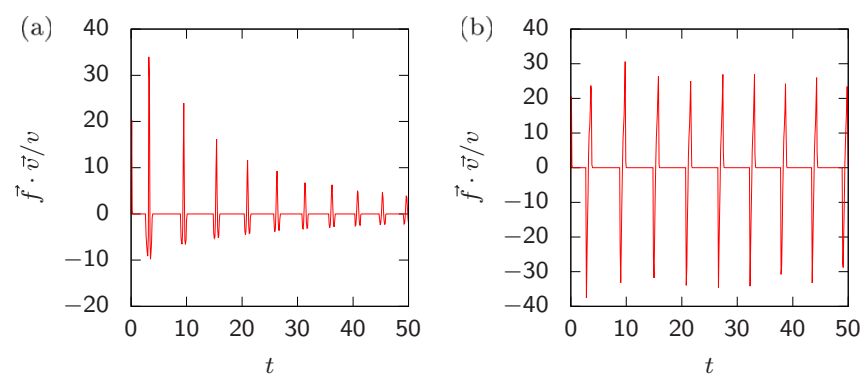

FIG. 13. Component of the total force $\vec{f}$ pointing in the direction of $\vec{v}$ as a function of $t$ for the simulation shown in (a) Fig. 14(a), and (b) Fig. 14(c). In (a), $\vec{f}$ tends to point at the direction of $\vec{v}$, whereas in (b) $\vec{f}$ points at the both directions of $\vec{v}$ and $-\vec{v}$ equally. 

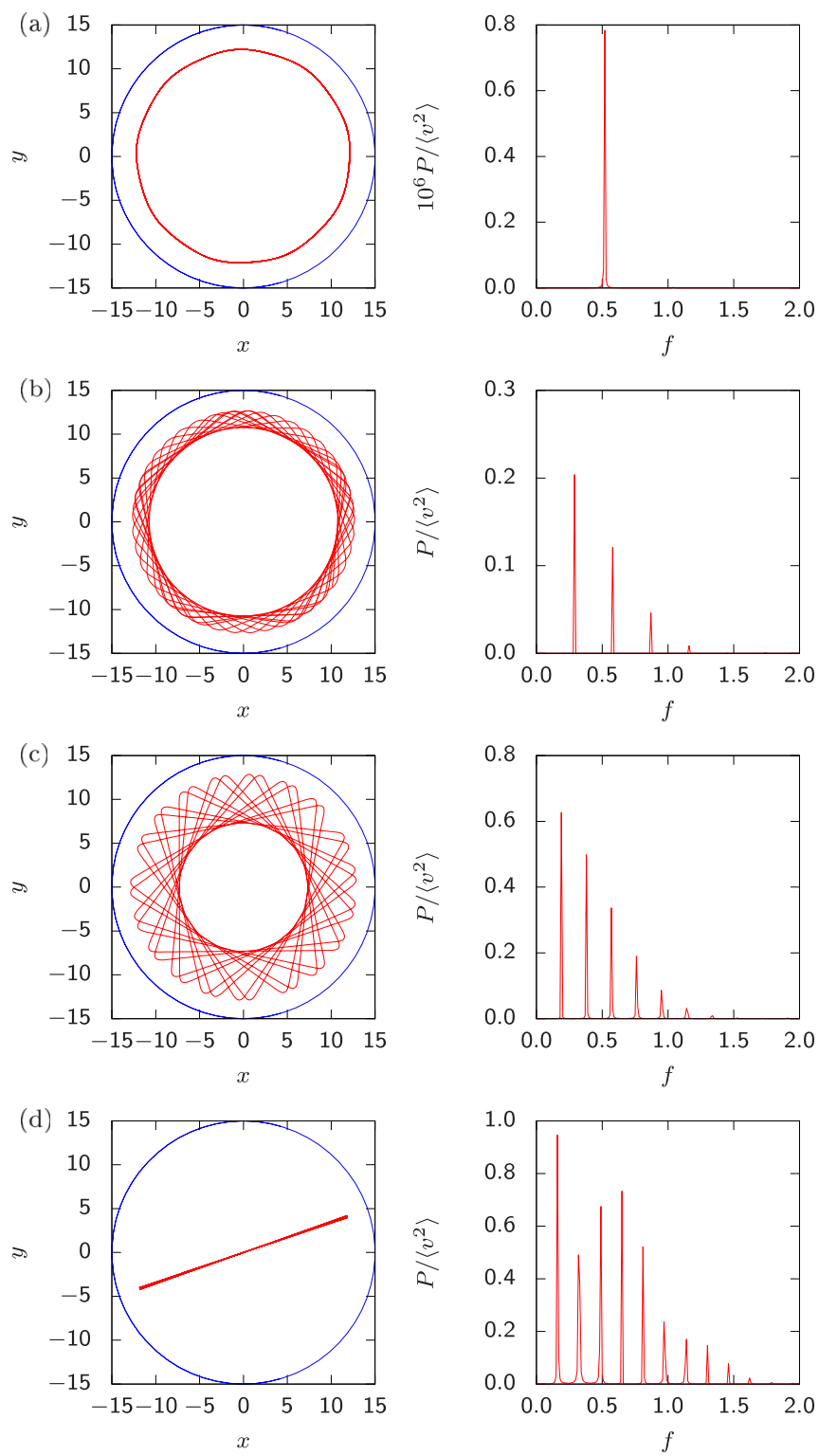

FIG. 14. Simulated trajectories (left column) and the power spectra of $v-\langle v\rangle$ (right column), based on Eq. (9). The force was fixed at $a_{d}=115$. (a) $b=1.0$, (b) $b=0.0$, (c) $b=-1.0$, and (d) $b=-4.5$. 